\title{
Educación ambiental para el manejo de los desechos sólidos hospitalarios en Jalapa El caso de los Centros de Atención Permanente -C.A.P.-
}

\author{
Yessenia Eunice Recinos Méndez \\ Médico y Cirujano con especialidad en Ginecología y Obstetricia \\ Maestra en Educación y Ambientalización Curricular \\ Área de Salud de Jalapa, Ministerio de Salud Pública y Asistencia Social \\ yessenia_recinos@yahoo.es
}

Fecha de recepción: 09/08/2014

Fecha de aceptación: 02/10/2014

\begin{abstract}
Resumen
En Guatemala en el año de 2001 se aprobó el reglamento para el manejo de desechos sólidos hospitalarios según Acuerdo Gubernativo No. 509-2001, que fue creado con el fin de velar por el mantenimiento del equilibrio ecológico y la calidad del medio ambiente, así como la de mejorar la calidad de vida de los habitantes del país, también cuenta con normas que tienden a la protección, establecimiento de acciones de prevención, regulación y control de las actividades que causan deterioro y contaminación. Sin embargo, para que este reglamento sea funcional, se necesita que el personal que presta servicios de salud en el país estén lo suficientemente educados y preparados. No obstante, hasta el momento no se tiene la certeza que se esté educando a dicho personal principalmente en los Centros de Atención Permanente denominados -C.A.P.-, por lo que la presente investigación pretendió evaluar el proceso formativo de los médicos, paramédicos, laboratoristas y conserjes sobre el manejo de los desechos hospitalarios lo que permitió obtener información básica para confirmar el cumplimiento del reglamento implementado por el ministerio de Salud Pública y Asistencia Social en el país que conlleva a minimizar la contaminación ambiental.
\end{abstract}

Palabras clave: Desechos sólidos hospitalarios, equilibrio ecológico, contaminación ambiental, educación ambiental, salud pública

\begin{abstract}
In Guatemala in 2001, the regulations for the management of hospital solid waste was approved by Governmental Resolution No. 509-2001, which was created in order to ensure the maintenance of ecological balance and environmental quality and improve the quality of life of the inhabitants of the country, also has provisions designed to protect, establishment of prevention, regulation and control of activities that cause deterioration and pollution. However, for this regulation is functional, it is necessary that the personnel providing health services in the country are sufficiently educated and trained. So far there is no certainty that such staff is educated mainly in Permanent Care Centers -CAP- called, so the present study aimed to evaluate the learning process of doctors, paramedics, laboratory technicians and janitors on the management of hospital waste allowing basic information to confirm compliance with the rules implemented by the Ministry of Health and Welfare in the country leading to minimize environmental pollution.
\end{abstract}

Key words: Hospital solid waste, ecological balance, environmental pollution, environmental education, public health 


\section{Introducción}

El manejo de la basura es un problema que aqueja a Guatemala cada vez más y se ha visto que uno de los principales factores que contamina al ambiente es la enorme cantidad de desechos sólidos que producen el comercio, la industria, las residencias familiares y los servicios de salud entre otros.

Desde los años setenta, se ha promovido a nivel mundial la importancia de dar un adecuado manejo a los desechos sólidos, ya que esto es uno de los principales problemas ambientales y sociales, sobre todo los materiales que no tienen uso directo y se descartan en forma permanente porque no se pueden reutilizar o reciclar (Koning, et al., 1994). Entre este tipo de desechos están los producidos por los servicios de salud que son los más contaminantes, y aunque algunos pueden manejarse con el resto de desechos de la población en general, otros necesitan de manejos especiales durante su eliminación. Los desechos producidos por los servicios de salud son también conocidos como desechos hospitalarios y se definen como todos aquellos generados durante el proceso de atención de salud tanto público como privado donde se practiquen los niveles de atención humana y veterinaria, con fines de prevención, diagnóstico, tratamiento, recuperación y rehabilitación de la salud (MSPAS, 2001).

\section{Definición del problema}

Los desechos generados por los establecimientos de atención de salud pueden presentar un riesgo para las personas y el medio ambiente, tanto dentro, como fuera de estos. Los desechos de establecimientos de salud, algunas veces se manejan como desechos domésticos, lo que puede representar un riesgo directo para los trabajadores de salud, los recolectores de basura y la población en general (Koning, et al., 1994).

Por lo tanto, para evitar dicha contaminación el Ministerio de Salud Pública y Asistencia
Social de Guatemala creó el Protocolo y Normas de Manejo de los Desechos Sólidos Hospitalarios en el año 2001 (MSPAS, 2001) y esta disposición hizo obligatorio que el personal médico, paramédico, laboratoristas y conserjes cuenten con la educación ambiental necesaria sobre la manipulación, clasificación y disposición de los desechos hospitalarios que permitan su manejo adecuado para evitar la contaminación del área de trabajo y del ambiente en general.

En el caso de los hospitales, los desechos sólidos están siendo manejados por empresas privadas y además se cuenta con incineradores dentro de las mismas instituciones. Sin embargo, a nivel de los Centros de Atención Permanente del área de salud de Jalapa, no se tiene la certeza sobre una educación apropiada a su personal para el buen manejo de los desechos sólidos lo cual puede llevar a inadecuadas prácticas repercutiendo en la contaminación de las personas y del ambiente en general.

El Área de Salud de Jalapa, cuenta con un total de 4 Centros de Atención Permanente conocidos como C.A.P. en los municipios de San Carlos Alzatate, San Pedro Pínula, Mataquescuintla y la aldea Sanyuyo en el municipio de Jalapa, en los cuales por tener pacientes internados, existe mayor producción de desechos hospitalarios y hasta el momento no se tiene ningún estudio que indique si estos desechos están manejándose adecuadamente y si el personal que labora en dichas instituciones está siendo educado al respecto.

Por lo tanto, es necesaria una investigación que permita establecer la forma en que se están manejando los desechos hospitalarios lo cual permitirá disminuir la contaminación para evitar riesgos a la salud de las personas y el deterioro del medio ambiente en general.

\section{Justificación}

El manejo inadecuado de los desechos peligrosos generados en los establecimientos de salud es un factor de riesgo para la transmisión de enfermedades, no sólo para 
la población que se atiende o labora en dichos centros, sino también para la población aledaña y el medio ambiente (Leal, et al 2004). A nivel nacional, el Ministerio de Salud Pública y Asistencia Social es el ente rector de la salud y lleva el liderazgo en todos los procesos definidos en las políticas de salud del gobierno (OPS, 2007).

Desde el año 2001 el Ministerio de Salud Pública y Asistencia Social estableció el Protocolo de Manejo de Desechos Sólidos Hospitalarios y su normativo, por lo que en todas las instituciones de salud; puestos de salud, centros de salud, centros de atención permanentes, hospitales, clínicas privadas y sanatorios, se debe dar a conocer dicho protocolo para evitar la contaminación ambiental.

En el departamento de Jalapa actualmente no se cuenta con ningún estudio en el cual se pueda evaluar el conocimiento y educación ambiental que los trabajadores de salud tienen sobre el manejo de desechos hospitalarios. La población que es atendida en el área de salud de Jalapa ha crecido por lo cual los desechos hospitalarios también han aumentado.

Entre los años 1974 y 1976 inició el área de salud de Jalapa contando únicamente con un Centro de Salud y con un Hospital Nacional, y debido a que actualmente la población es mayor se ha tenido la necesidad de incrementar los centros de atención teniendo hoy en día 8 centros de salud, un hospital nacional además de varios puestos de salud, por lo cual es importante determinar con qué frecuencia se educa al personal sobre el manejo de los desechos hospitalarios, para evitar la contaminación de la población y del medio ambiente en general.

En la siguiente investigación se tomó como objeto de estudio a los Centros de Atención Permanente de Jalapa conocidos como C.A.P. ya que es en estos donde se encuentran un mayor número de personal y son las áreas con mayor producción de desechos hospitalarios, siendo las instituciones que tienen mayor riesgo de contaminación.

\section{Objetivos}

\section{General}

Evaluar la formación ambiental del personal de los Centros de Atención Permanente -C.A.P- de Jalapa sobre el manejo de desechos hospitalarios.

\section{Específicos}

Evaluar el conocimiento del protocolo de manejo de desechos hospitalarios en los centros de atención permanente del departamento de Jalapa.

Establecer la aplicación de las normas para el manejo, clasificación y destino de los desechos hospitalarios.

Identificar la necesidad de educación sobre el manejo de desechos hospitalarios en el personal que labora en los Centros de Atención Permanente en el departamento de Jalapa.

Propiciar la educación ambiental continua como estrategia en el manejo de desechos sólidos hospitalarios en los trabajadores de los Centros de Atención Permanente de Jalapa.

\section{Hipótesis}

El personal médico, paramédico, laboratoristas y operativo de los Centros de Atención Permanente -C.A.P.- del departamento de Jalapa en Guatemala, no cuenta con la formación ambiental apropiada sobre el manejo de los desechos sólidos hospitalarios.

\section{Antecedentes}

El Ministerio de Salud Pública y Asistencia Social es el ente regulador de la salud a nivel nacional. Dentro de las obligaciones del 
Ministerio, a través de sus áreas de salud, es velar que se cumpla el adecuado manejo de los desechos hospitalarios.

En el año 2001 se aprobó el protocolo de manejo de desechos sólidos hospitalarios según Acuerdo Gubernamental 509-2001 con su respectivo normativo, siendo el Ministerio de Salud el encargado de velar porque dicho protocolo se cumpla en todas las instituciones de salud tanto públicas como privadas. El Ministerio de Salud se divide en áreas en cada departamento de Guatemala teniendo diferentes niveles de atención, siendo estas:

a) Primer nivel de atención: Centros comunitarios de salud que son los establecimientos de menor complejidad de la red de servicios como los centros de convergencias, y puestos de salud.

b) Segundo nivel de atención: Conocidos como Centros de Salud que se encuentran ubicados en el ámbito municipal y generalmente en las cabeceras municipales, regulando y coordinando los servicios de menor categoría, es decir a los del primer nivel, brindando servicios de promoción, prevención, recuperación y rehabilitación dirigidos a las personas y acciones al ambiente. Los Centros de Salud se clasifican en: Centros de Salud Tipo A, Centros de Salud Tipo B, Centro de Atención a Pacientes Ambulatorios (CENAPA), Centro de Atención Médica Permanente (CAP), Centro de Atención Integral Materno-Infantil (CAIMI), y otros Centros de Salud como Clínicas Periféricas, Centros de Urgencia Médicas (CUM) y Maternidades Periféricas.

c) Tercer nivel de atención: Hospitales departamentales, regionales y de referencia, que tienen una mayor complejidad $y$ capacidad resolutiva (MSPAS, 2014).

Según el Manual de Organización, procedimientos y normas del personal de salud de Jalapa (2006), el Área de Salud de Jalapa fue fundada entre los años 1974 y 1976 y actualmente tiene a su cargo 8 centros de Salud, uno en cada cabecera municipal; Jalapa, Monjas, San Luis
Jilotepeque, San Manuel Chaparrón, Mataquescuintla, San Carlos Alzatate, San Pedro Pínula y uno en la aldea Sanyuyo, siendo los últimos cuatro Centros de Atención Médica Permanente conocidos por sus siglas como -C.A.P.-.

Cada centro de Atención Permanente atiende a pacientes todos los días de la semana en horario continuo, teniendo encamamiento para la atención de partos. En los Centros de Atención Permanente de Mataquescuintla, San Pedro Pínula y Sanyuyo además cuentan con laboratorios clínico en los cuales se procesan exámenes de sangre, heces, orina y esputo ${ }^{1}$ entre otros. Cada uno de los centros de Atención Permanente debe de estar cumpliendo con el protocolo de manejo de desechos sólidos hospitalarios.

\section{Metodología}

La metodología de la investigación fue de tipo mixto, cuantitativo y cualitativo que consistió en determinar si el personal en los C.A.P. del departamento de Jalapa en Guatemala tenía una formación ambiental sobre el manejo de los desechos hospitalarios.

Para el efecto se realizaron entrevistas al personal por estratos en los que se encuentran los médicos, enfermería o paramédicos, técnicos de laboratorio y operativos o conserjes. Se utilizó una guía de preguntas o cuestionario, como instrumento de recolección de información que se dirigió al personal de los C.A.P.

Paralelamente a la recolección de información se desarrolló un taller participativo en cada centro de atención permanente con el objetivo de establecer si el personal está aplicando las normas para el manejo, clasificación y destino de los desechos hospitalarios así como identificar la necesidad que tiene dicho personal de educarse $y$ capacitarse en el manejo de desechos hospitalarios. 
Finalmente se buscó implementar una estrategia para la educación continua en el manejo de desechos sólidos hospitalarios entre los trabajadores de los C.A.P. de Jalapa, se realizó una reproducción del Acuerdo Gubernativo 509-2001 de forma masiva y se les compartió a to el personal, dando instrucciones de la forma apropiada de utilizarlo.

\subsection{Método de investigación}

El método que se utilizó fue el estudio de caso con enfoque de investigación acción debido a que se analizó el proceso de educación que se realiza en los Centros de Atención Permanente ubicados en los municipios de San Carlos Alzatate, San Pedro Pínula, Mataquescuintla y la aldea Sanyuyo en el municipio de Jalapa, y luego de obtener el diagnóstico y la información se realizó un taller de capacitación para dar a conocer las normas y regulaciones sobre el manejo de los desechos hospitalarios en los cuatro C.A.P. en estudio.

\subsection{Técnicas de investigación}

\section{a) Observación}

Esta técnica, se empleó mediante recorridos y visitas en los C.A.P. para verificar si el personal médico, paramédico, laboratoristas y operativo realizan la manipulación, clasificación y disposición de los desechos hospitalarios de forma apropiada.

\section{b) Talleres Participativos}

Se realizó un taller participativo con el personal en cada C.A.P para socializar con los médicos, paramédicos, laboratoristas y personal operativo, el protocolo y normas del ministerio de Salud Pública y Asistencia Social de Guatemala sobre el manejo de desechos sólidos, con la finalidad de evitar la contaminación ambiental.

\section{c) Entrevistas}

Las entrevistas se realizaron al personal por estratos entre los cuales estuvieron los médicos, enfermería o paramédicos, técnicos de laboratorio y operativos o conserjes, con los que se utilizó una guía de preguntas o cuestionario como instrumento de recolección de información.

\subsection{Instrumentos utilizados en la investigación}

Para la recopilación de información se empleó una serie de cuestionarios dirigidos al personal de los C.A.P. que se estratificaron según su nivel educativo y se agruparon en médicos, enfermería o paramédicos, técnicos de laboratorio y operativos o conserjes.

\section{Marco teórico}

\subsection{Desechos sólidos}

Se considera desecho a cualquier material a partir del momento en que haya sido descartado y que ya no tiene ninguna utilidad, generándose a partir de la vida diaria. En el caso de los residuos sólidos urbanos, provienen de las actividades que se desarrollan en el ámbito doméstico, comerciales, industriales y de servicios. Estos están compuestos por residuos orgánicos (excedentes de comida y restos de material vegetal), materiales reciclables como papel, cartón, madera e inorgánicos como, vidrio, plástico, metales, etc. (Plaza y Zapata, 2011).

La producción de residuos urbanos y asimilables a urbanos se ha incrementado en forma considerable en los últimos años, siendo esta una tendencia general (Díaz, 2007). Dichos desechos han aumentado debido a la generación de residuos municipales, convirtiéndose esto en un problema especialmente para los países en vías de desarrollo (Vásquez, 2011).

Según Lacayo (2008), los residuos sólidos se han vuelto un problema común en la región centroamericana, sin tener la infraestructura adecuada y los recursos tanto a niveles municipales, gubernamentales 0 en instituciones encargadas de dichos desechos. En Guatemala anualmente se produce una generación de 81203,153 toneladas de basura, contribuyendo a aumentar el 
problema de contaminación por residuos sólidos (URL, 2003).

Los desechos sólidos acumulados son propicios para formar refugios de diversos vectores ya que crean condiciones propias para poder reproducirse y propagarse, llegando a ser contaminantes para las poblaciones y el ambiente en general (Plaza y Zapata, 2011). Son producidos por toda la población no quedando fuera de ello los servicios de salud donde se producen desechos sólidos tanto comunes como hospitalarios por lo cual en la gestión del manejo de desechos sólidos es importante la participación de todo el personal en la separación y la recolección de dichos residuos, teniendo un convencimiento personal sobre la importancia que esto lleva para tener un impacto ambiental favorable, debiendo mejorar las estrategias existentes (Ruiz, 2012).

En 1982 el hallazgo de residuos médicos en las playas del estado de New Jersey, E.U.A., llevaron la aprobación de la ley que se denominó Ley de Control de Residuos Médicos, a partir de la cual comienzan a crearse procedimientos para la identificación y separación de los residuos. Sin embargo, no es hasta que la ONU en 1992 realiza la conferencia sobre el medio ambiente $y$ desarrollo en Río de Janeiro, que empiezan a gestionarse estrategias a nivel mundial para el manejo integral de los desechos hospitalarios debido a que en los establecimientos de salud constituye un factor de riesgo importante para la trasmisión de enfermedades, no sólo para la población que atiende o labora en dichos centros, sino también para la población aledaña y el medio ambiente (Leal et al., 2004).

\subsection{Desechos hospitalarios}

Según el Acuerdo Gubernativo No. 5092001 del Ministerio de Salud Pública y Asistencia Social de Guatemala, define a los desechos hospitalarios como aquellos que producen los entes generadores durante el desarrollo de las diferentes actividades es decir, los producidos por todos los establecimientos en los cuales se practique la atención humana o veterinaria con fines de prevenir, diagnosticar, dar tratamiento, promover la recuperación y rehabilitación de la salud en los hospitales públicos y privados, clínicas médicas y veterinarias, laboratorios clínicos, clínicas odontológicas, maternidades, casas maternas, centros de salud, centros de atención permanente, etc.

Los residuos generados por los centros hospitalarios pueden causar problemas asociados por lo cual han sido motivo de preocupación internacional. Los desechos biomédicos producidos en las diferentes actividades de la atención en salud tienen un alto potencial infeccioso más que cualquier otro desecho sólidos (Katoch 2007), aunque un buen porcentaje no producen riesgo infeccioso, por lo cual se pueden comparar con los desechos domésticos o comunes (Pruss et al., 1999), pero el resto comprenden un alto grado de propagación de enfermedades infecciosas 0 hasta riesgo ambiental derivados de los métodos empleados para su tratamiento y disposición final (Neveu y Matus, 2007).

Los desechos generados en hospitales y centros de atención en salud representan "dificultades y riesgos especiales por el carácter infecciosos de algunos de sus componentes por su composiciones heterogéneas, la presencia de objetos punzocortantes y eventualmente sustancias tóxicas, inflamables y radiactivas de baja intensidad" (Junco y Rodríguez, 2002:122). El manejo deficiente de los desechos hospitalarios puede crear, no sólo situaciones que pongan en riesgo la salud de la población que se encuentra en los diferentes centros de atención en salud, es decir personal y pacientes, sino puede causar también situaciones de deterioro ambiental que llegue más allá de los límites del centro asistencial, sometiendo a riesgo la salud de la comunidad directa o indirectamente.

Debido a la variedad de los desechos hospitalarios, es de vital importancia clasificarlos adecuadamente para poder darles un tratamiento especial y así poder 
evitar la contaminación de la población en general. Según el Decreto 509 - 2001, los desechos hospitalarios se clasifican en desechos hospitalarios bioinfecciosos, desechos hospitalarios especiales, desechos hospitalarios comunes y otros desechos.

\subsubsection{Desechos hospitalarios bioinfecciosos}

Son lo que se generan durante las diferentes etapas de la atención en salud y que han entrado en contacto con pacientes humanos o animales y presentan diferentes niveles de peligrosidad de acuerdo al grado de exposición que hayan tenido con los diferentes agentes infecciosos que causan enfermedades.

Estos desechos pueden ser:

a) Materiales provenientes de aislamiento de pacientes

b) Materiales biológicos

c) Sangre humana y producto de sus derivados, incluyendo los recipientes utilizados para su obtención o manejo

d) Desechos anatómicos patológicos y quirúrgicos

e) Desechos punzocortantes

f) Desechos animales

\subsubsection{Desechos hospitalarios especiales}

Son todos aquellos desechos que se generan durante las actividades auxiliares en los centros de atención en salud, pero que no han estado en contacto directo con los pacientes ni con agentes infecciosos, pueden constituir un peligro para la población por su carácter agresivo.

En este grupo de desechos se pueden mencionar:

a) Desechos químicos peligrosos que son todos aquellos provenientes de productos utilizados para diagnóstico, quimioterapia, trabajos experimentales, limpieza y desinfección.

b) Desechos farmacéuticos como medicamentos vencidos no utilizados, contaminados, etc. c) Desechos radiactivos que son materiales contaminados con radionúcleos de baja actividad provenientes de laboratorios químicos, biológicos, de análisis clínicos y servicios de medicina nuclear. Los desechos radiactivos con actividad media o alta deben de disponerse en lugares especiales mientras su actividad baja hasta que ya se lleguen a los límites permitidos para su eliminación.

\subsubsection{Desechos hospitalarios comunes}

Son aquellos que se generan en las actividades administrativas, auxiliares y generales que no representan peligros para la salud y sus actividades, son similares a los que presentan los desechos domésticos comunes, es decir materiales de oficinas, restaurantes o desechos de los pacientes que no representan riesgos infecciosos. Platos desechables, etc.

\subsubsection{Otros desechos}

Se incluyen todos aquellos equipos no utilizados u obsoletos.

\subsection{Manejo de desechos hospitalarios}

El manejo de los desechos sólidos generados en instituciones de salud es uno de los aspectos de la gestión hospitalaria que han ido tomando importancia e interés ya que esto puede llevar consigo mucha contaminación, constituyendo un problema de salud pública, saneamiento ambiental, enfermedades nosocomiales, epidemiológicos y otros (Carranza, 2002). "Estos desechos considerados peligrosos por sus características pueden ser: tóxicos, explosivos, inflamables, corrosivos e infecciosos" (Junco y Díaz, 2000:195) y por su heterogeneidad pueden aumentar la dificultad de su manejo.

El manejo deficiente de los desechos hospitalarios puede amenazar la salud de la población hospitalaria como el personal y pacientes, creando situaciones de riesgo, así como deterioro ambiental al trascender los límites de los centros de atención en salud poniendo en riesgo a la población cercana a dichos centros y las personas que los 
manipulan. El establecimiento de un plan de manejo de los desechos brinda y minimiza los riesgos a la salud de los involucrados. Existen diferentes etapas en el manejo de los desechos sólidos hospitalarios por lo cual deben de implementarse las medidas adecuadas para prevenir cualquier riesgo que pueda derivarse de la manipulación de estos desechos (Suarez y Junco, 2012). El ministerio de Salud y Asistencia Social de Guatemala establece que toda institución generadora de desechos hospitalarios debe de incluir dentro del plan de manejo las siguientes etapas:

a) Separación y embalaje

b) Almacenamiento en cada unidad de generación

c) Recolección y transporte intrahospitalario

d) Almacenamiento Intrahospitalario

e) Transporte extra hospitalario

f) Tratamiento

g) Disposición final

Deben de ser separados todos los desechos producidos en las instituciones de salud para lo cual es necesario tener recipientes bien identificados y embalados, los cuales deben de ser de fácil manejo y que el material no sea susceptible a roturas para evitar que se derramen.

- Clasificación para la separación y embalaje:

- Los desechos infecciosos deberán de colocarse en recipientes o bolsas de color rojo con la simbología de bioinfeccioso, los materiales punzocortantes deberán ser destruidos por maquinas trituradoras o termo trituradoras o en cajas de cartón o recipientes plásticos adecuados y debidamente sellados, debiendo de colocarse dentro de las bolsas de color rojos 0 introducidos en las trituradoras o termo trituradoras.

- Los desechos especiales deben de colocarse en Bolsas Blancas con la simbología de químicos. Los recipientes de cristal entero o roto deben de colocarse en cajas de cartón o recipientes plásticos adecuados y debidamente sellados, debiendo depositarse dentro de las bolsas de color blanco.

- Los desechos radiactivos en general deben de colocarse en contenedores de plomo adecuados al nivel de radiación que les corresponda, debidamente identificados con la simbología de radiactivos, estos desechos van separados ya que corresponden al ministerio de energía y minas la orden de su disposición final.

- Los desechos comunes deben de depositarse en bolsas o envases de color negro.

La simbología que deberá utilizarse para cada bolsa de almacenaje según el Acuerdo Gubernativo 509-2001 del Ministerio de Salud Pública y Asistencia Social de Guatemala se muestra en la figura 1:

Figura 1 Simbología a utilizar en cada bolsa de almacenaje

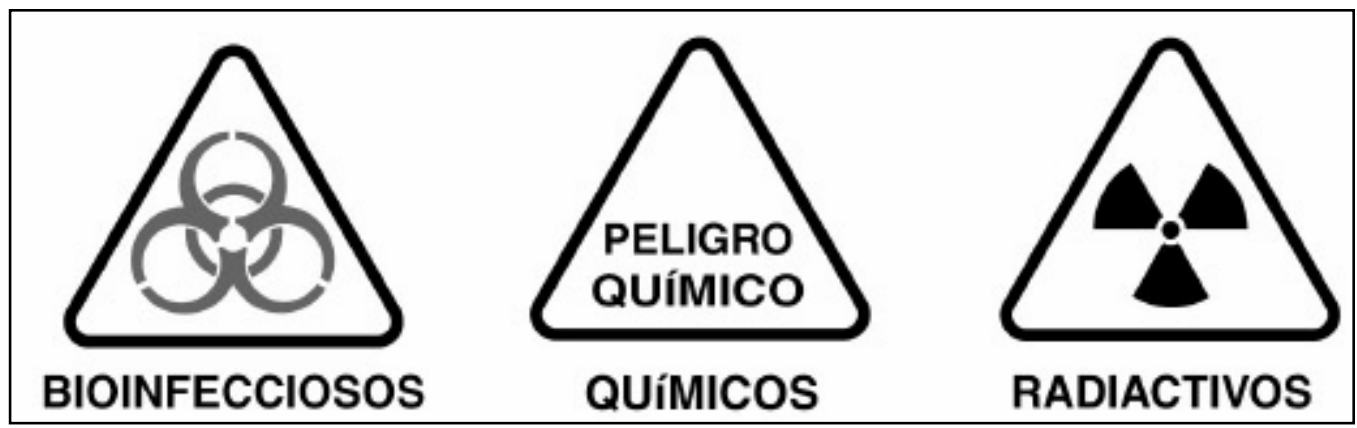

Fuente: Acuerdo Gubernativo 509-2001 
Las características de dichas bolsas deberán ser bolsas de polietileno de baja densidad con agregado de resina (AR) industrial con espesor mínimo de 250 a 300 micras con dimensiones de 0.5 a 0.9 metros con cierre hermético 0 cualquier otro dispositivo aprobado por el departamento de Salud y Ambiente, el embalaje deberá de llevar la siguiente información:

a) Nombre de la institución generadora

b) Fecha y hora de su recolección

c) Procedencia interna de la institución

d) Operador responsable

e) Hora de recepción en el lugar temporal de almacenamiento en la institución

f) Fecha y hora de salida para su tratamiento (en desechos infecciosos)

g) Fecha y hora de salida para su disposición en el relleno sanitario (en desechos hospitalarios especiales)

h) Fecha y hora de salida para su disposición dentro del sistema que el ministerio de energía y minas establezca (en desechos radiactivos)

Cada unidad generadora de desechos sólidos hospitalarios debe de tener depósitos adecuados necesarios para cubrir el volumen de la cantidad producida y de conformidad con la naturaleza de los mismos.

Los desechos generados en las diferentes actividades de la atención en salud deberán ser colocados en recipientes que tengan las siguientes características: estar claramente identificados y contar con la simbología específica, encontrándose en buenas condiciones físicas, sin corrosión ni vaciamiento, siendo de material compatible con el material que contendrán y estar siempre cerrados excepto en el momento de llenado y vaciado, dichos recipientes deberán de colocarse en lugares con superficies impermeables y preparados para impedir la percolación en caso de derrame. Los depósitos temporales que se tengan en cada unidad generadora deben de separar físicamente los diferentes tipos de desechos.

Es importante que cada institución cuente con una recolección periódica de los desechos y estos deben de ser llevados a un centro temporal interno donde puedan estar de forma segura para no contaminar, los desechos deben de ser manejados por personal capacitado el cual cumpla con los requerimientos necesarios de protección personal. El transporte externo debe de realizarse en vehículos cerrados que cuenten con personal entrenado para su transporte, cada Área de Salud es responsable de la vigilancia del transporte de los desechos hospitalarios.

En estudio realizado sobre la disposición final de los desechos sólidos hospitalarios en el Hospital regional de occidente "San Juan de Dios" de Quetzaltenango, se concluye que la capacitación al personal es importante y que a corto plazo es preferible la contratación de una institución privada que aunque a largo plazo es más cara, pero a corto plazo resuelve el problema de la contaminación al no estar tratando adecuadamente los desechos (Ramirez, 2005). En el hospital Roosevelt también se realizó un estudio sobre la factibilidad para la implementación de un sistema de tratamiento de desechos sólidos hospitalarios (De Leon, 2011), y se concluye que el buen funcionamiento en el manejo se basa en la capacitación que se tiene en el recurso humano que se encarga del manejo de dichos desechos teniendo una cultura adecuada en el tren de aseo tanto interno como externo, con lo cual se sigue confirmando que la Educación ambiental es importante en el adecuado manejo de estos desechos. Establecer planes de capacitación con el fin de concientizar sobre el manejo de los desechos sólidos buscando enfatizar el cuidado de la salud humana y protección de medio ambiente debe de realizarse en cada centro de atención en salud.

Para Rodríguez (2006), el personal involucrado en el manejo de desechos sólidos hospitalarios debe de ser capacitado todo ya que este es un personal rotativo y son quienes están más expuestos a accidentes laborales constituyendo en un riesgo de contaminación mayor. 


\subsection{Recolección y transporte intrahospitalario}

Este proceso debe de llenar los siguientes requisitos:
a) Recolección y transporte intra- hospitalario
b) Almacenamiento intrahospitalario
c) Transporte extrahospitalario.
d) Tratamiento
e) Disposición final.

\subsection{Educación ambiental para el manejo de desechos hospitalarios}

Según Mata (2006), la educación ambiental es un instrumento práctico para lograr resultados cuando se necesita producir un cambio en las formas de uso del ambiente.

En la declaración de Rio de Janeiro sobre el Medio Ambiente y el Desarrollo realizada por Naciones Unidas en junio de 1992, el principio 10 sugiere que

el mejor modo de tratar las cuestiones ambientales es con la participación de todos los ciudadanos interesados, en el nivel que corresponda. En el plano nacional, toda persona deberá tener acceso adecuado a la información sobre el medio ambiente de que dispongan las autoridades que encierran peligro en sus comunidades, así como la oportunidad de participar en los procesos de adopción de decisiones. Los estados deberán facilitar y fomentar la sensibilidad y la participación de la población poniendo la información a disposición de todos. Deberá proporcionarse acceso efectivo a los procedimientos judiciales y administrativos, entre estos el resarcimiento de daños y los recursos pertinentes (ONU, 1992:4).

La educación ambiental es un proceso de formación permanente que no debe de excluir a nadie, relaciona lo individual con lo colectivo, involucrando actitudes y valores de los individuos a las comunidades, debiendo tener una transformación en los sistemas educativos para tener un ambiente sano y ecológicamente equilibrado (Zambrano, 2009).

La educación ambiental debe darse en todas las instituciones de salud, tomando conciencia que determinados desechos hospitalarios pueden llevar riesgos para la salud pública por lo cual los programas de capacitación periódica para todo el personal interdisciplinario que labora en las instituciones de salud es importante, dando capacitaciones sobre bioseguridad, y los protocolos existentes sobre el manejo de desechos hospitalarios (Rodríguez y Trindade, 2010).

La temprana acción sobre la identificación de los factores de riesgos ambientales que afectan la salud, y la búsqueda de soluciones a nivel local donde se involucre a la comunidad y a otros sectores, hará posible que algunos de los problemas del medio ambiente sean resueltos con la participación de todos (Mulet, 2002).

El manejo integral de los desechos hospitalarios implica el desarrollo adecuado de cada una de las etapas de generación, separación, desactivación, almacenamiento, recolección, transporte, tratamiento y disposición final de ellos, al no realizarse estos procesos ya sea por desconocimiento o negligencia por parte de las personas involucradas pueden presentarse impactos negativos que afecten la salud humana y el ambiente, generando problemas sociales, ambientales y económicos.

La educación del personal que labora en las diferentes instituciones de salud sobre el manejo de desechos hospitalarios es importante ya que al darles a conocer el tratamiento correcto de ellos evitará la contaminación de las áreas de trabajo y de la población en general. Trabajar para minimizar la cantidad y toxicidad de toda la basura generada por el sector salud es importante de tal manera que el manejo de los residuos se haga protegiendo a las personas y el ambiente (Rodríguez y Trinidade 2010), por lo cual, la clasificación adecuada de los desechos hospitalarios 
permite derivar a los desechos especiales y de alto riesgo a una recolección especial y los desechos comunes junto con los desechos municipales (Junco y Díaz, 2000).

La educación ambiental debe impartirse en todos los servicios de salud desde el primer nivel hasta el tercer nivel, es decir desde los pequeños puestos de salud hasta los grandes hospitales. Es imprescindible que el tema sea manejado por todo el personal que labora en dichas instituciones ya que con frecuencia sucede que es de interés exclusivo de los directivos y de algún profesional con inquietudes respecto al cuidado del medio ambiente (Salud sin daño, 2007).

La educación y capacitación continua a los trabajadores de salud es importante sobre todo en la bioseguridad, siendo esto el conjunto de medidas y disposiciones técnicas las cuales van orientadas a la protección humana y ambiental entre otras (Hernández, et al., 2006).

El manejo seguro de los desechos hospitalarios requiere la diligencia y el cuidado de todas las personas involucradas con el fin de disminuir los riesgos y de mejorar sus condiciones de trabajo ya que en gran medida las malas prácticas del manejo de estos desechos son consecuencia de no dar educación al personal encargado (Mata, et al., 2006).

Utilizando los métodos correctos para desarrollar los diferentes trabajos en las áreas de salud, es decir usar los equipos de protección personal, tomar medidas preventivas apropiadas para intervenir equipos, identificar los riesgos existentes en las áreas de trabajo constituye precauciones fundamentales en torno al trabajo digno, seguro y saludable, por lo cual se debe de incorporar las normas y procedimientos que garanticen un desempeño seguro $y$ anticiparse a cualquier situación de riesgo debiendo capacitarse a cada trabajador es decir trabajar en el saber (proveer el conocimiento) saber hacer (trabajar las habilidades) y saber ser/actuar (la prevénción y seguridad) (ISL 2011).
La sensibilización y capacitación que se dirige al personal laboral en salud con el propósito de dar a conocer los aspectos relacionados en el manejo integral de los residuos debe de incluir los procedimientos específicos con que se maneje cada desecho, las funciones y responsabilidades que cada persona tiene, los mecanismos de coordinación y las directrices de cada institución (Área metropolitana de Aburrá, 2008).

Toda persona que ingresa a trabajar a las instituciones de salud debe de conocer el Manual de Manejo de desechos hospitalarios, a fin de generar una cultura ambiental y facilitar el manejo de los desechos minimizando así los posibles impactos negativos en el ambiente y la salud (INS 2010), los programas de formación y educación deben de contemplar estrategias y metodologías de sensibilización y capacitación (UTP 2006), de acuerdo al trabajo que cada uno vaya a desempeñar.

Se debe de concientizar a los trabajadores de salud que el primer principio de seguridad se refiere a proteger su propia vida, lo cual también implica responsabilidad con los que le rodean como su pareja, sus hijos, sus padres, sus amigos, sus compañeros y su comunidad (ISL, 2011).

\subsection{Efectos adversos del mal manejo de los desechos sólidos hospitalarios}

Los desechos hospitalarios representan un riesgo para la salud de los trabajadores, la comunidad y el ambiente (Larrañaga y Fernández, 2012), sobre todo, aquellos que contienen agentes patógeneos infectocontagiosos (García, 2010).

El interés sanitario y ambiental en relación a los desechos hospitalarios se ha incrementado a partir de los años 80, tras la aparición del VIH-SIDA, cuando se percibió como un problema potencial para la salud pública (Llorente, 1997).

Todas las personas que laboran en establecimientos de salud están expuestas a un grado variable de residuos peligrosos variando el riesgo según la permanencia en el 
establecimiento, las características de su trabajo y su participación en el manejo de dichos residuos, ya que estos residuos constituyen una categoría específica que, debido a su cantidad, concentración o características físicas, químicas o infecciosas, pueden a) causar un aumento de la mortalidad o un aumento de enfermedades graves irreversibles 0 reversibles que producen invalidez, 0 contribuir significativamente a ello, o b) plantear un riesgo sustancial real o potencial a la salud humana 0 al medio ambiente cuando son tratados, almacenados, transportados, eliminados o manejados en forma indebida (Koning 1994). En algunos estudios se ha determinado que los grupos más expuesto a estos riesgo son los trabajadores de las unidades de salud, especialmente las enfermeras y el personal de limpieza, incluyendo los trabajadores que manipulan los desechos hospitalarios fuera de las instalaciones (Hernández, et al., 2006).

Los desechos peligrosos pueden causar diferentes patologías en el ser humano por ejemplo: manifestaciones dermatológicas, ópticas, respiratorias, carcinogénicos, mutagénicos, teratogénicos, inmunológicos y otros (Sardiñas et al., 2001). Dichos problemas son especialmente graves en países en desarrollo en los cuales no se cuenta o es muy pobre la tecnología para el adecuado tratamiento y disposición final de los desechos.

El medio ambiente circundante se ve afectado contaminando los lugares cercanos donde se depositan dichos desechos o son eliminados inadecuadamente. En la mayoría de países de Latinoamérica y el Caribe se ha iniciado la consideración de aspectos institucionales y legislativos de los desechos peligrosos. Sin embargo, no se ha observado una infraestructura adecuada que posea los medios administrativos y tecnológicos necesarios para tratar las cantidades de desechos peligrosos que están generándose (Koning, 1994).
El mal manejo de los desechos hospitalarios puede llevar a la presencia de infecciones nosocomiales definiéndose como

las infecciones contraídas durante una estadía en el hospital que no se habían manifestado ni estaban en período de incubación en el momento del internado del paciente. $O$ las infecciones que ocurren más de 48 horas después del internado (OMS, 2002:4).

Por su parte, de acuerdo con Benenson (1995), las infecciones nosocomiales pueden ser aquellas que se presentan en un paciente internado en un hospital o en otro establecimiento de atención de salud en quien la infección no se había manifestado ni estaba en período de incubación en el momento del internado. Comprende las infecciones contraídas en el hospital producto de la contaminación ambiental existente, pero manifiestas después del alta hospitalaria y también las infecciones ocupacionales contraídas por el personal del establecimiento.

Los establecimientos de atención de salud son un entorno donde se congregan personas con diferentes patologías y están expuestas a mayores infecciones. Es por ello que el adecuado manejo de los desechos es de vital importancia para evitar la contaminación de las personas que asisten a los centros de atención en salud así como los trabajadores que laboran en ellas. Por lo que el medio ambiente en el cual se labora debe considerar las características de las instalaciones, es decir, el sistema de ventilación, el suministro de agua y alimentos y la evacuación de desechos en los centros de atención (OMS, 2002).

Los procesos que se desarrollan en el sector de salud están enfocados a mejorar la atención de los usuarios, es decir los pacientes, pero no debe de olvidarse que el personal que labora en dichas instituciones está expuesto a contaminación por lo cual, se debe de ofrecer un ambiente seguro que los resguarde de posibles lesiones que pueden 
ser causadas por manipulación de los desechos hospitalarios debiéndose a una inadecuada manipulación de objetos punzocortantes, 0 por el material contaminado con secreciones entre otras (Suarez y Junco 2012).

Aunque los desechos hospitalarios no son muy significativos en volumen frente a los residuos sólidos en general, sí revisten un grado importante de peligrosidad aumentando las enfermedades infecciosas que pueden adquirir los trabajadores de los centros de atención en salud (Palma 2008), y pueden tener consecuencias sobre la salud de las personas que cohabitan con ellos, por lo cual se debe de insistir en que todos los trabajadores utilicen los equipos de protección personal adecuados al tipo de riesgo que están afrontando, tomar las medidas preventivas apropiadas para ejecutar los trabajos, para intervenir los equipos e identificar los riesgos existentes en cada área constituyendo estas precauciones fundamentales en torno al trabajo, digno, seguro y saludable (ISL, 2011).

El ministerio de Salud de Chile (2001) informó que según evidencias epidemiológicas en Canadá, Japón y Estados Unidos han establecido que los desechos infecciosos de los hospitales han sido causantes directos de transmisión del agente del VIH y aun con mayor frecuencia los virus que trasmiten hepatitis B o C a través de las lesiones causadas por agujas y otros punzocortantes, aunque el riesgo es bajo se ha estimado que sólo el $1 \%$ de los accidentes con punzocortantes afecta a trabajadores hospitalarios no se puede desestimar el riesgo ya que el contagio después de una exposición de este tipo es en promedio de $0,3 \%$ para sangre contaminada con $\mathrm{VIH}$, $1,8 \%(0 \%-7 \%)$ para hepatitis $\mathrm{C}, \mathrm{y}$ entre $6 \%$ y $30 \%$ para hepatitis $\mathrm{B}$.

La diferencia se explica en parte por la mayor viabilidad de los virus de la hepatitis en el ambiente, y por la infectividad inherente de cada agente. De aquí la importancia de la bioseguridad la cual se define como el conjunto de normas y procedimientos que se realizan para proteger al personal de salud, a los pacientes y familiares de los pacientes que acuden a las instituciones de salud (Arze, 2005).

\subsection{Algunas enfermedades asociadas a la inadecuada gestión de residuos hospitalarios y similares.}

Gualdrón y Calderón (2012), indican que existe una gran cantidad de enfermedades asociadas con los residuos generados en instituciones de salud y similares, los cuales poseen un carácter peligroso, tanto desde el punto de vista infeccioso, radiactivo y químico. Desde el punto de vista infeccioso o de riesgo biológico los desechos hospitalarios pueden poseer, bacterias, virus, y otros organismos patógenos (Ministerio Salud de Colombia, 2002). Se debe tomar en cuenta que los virus pueden conservar su patogenicidad durante mucho tiempo en el ambiente.

\section{Resultados}

A través del trabajo de campo se pasó un cuestionario a un total de 65 trabajadores de los diferentes Centros de Atención Permanente (CAP) del Área de Salud de Jalapa, entrevistándose a médicos, enfermeras, laboratoristas y personal operativo (personal de limpieza y pilotos). Posterior a pasar las entrevistas se dio capacitación sobre el manejo de desechos sólidos hospitalarios con el fin de dar a conocer el Acuerdo Gubernativo 509-2001 donde se logró una comunicación de doble vía lo cual permitió complementar la información obtenida a través de los cuestionarios.

Para determinar si los trabajadores de los CAP tienen conocimiento si dentro de las instituciones de salud existen normas referentes a los desechos sólidos hospitalarios, se les realizó la pregunta para determinar si existen dichas normas y si estas son accesibles para todos, encontrándose que el $20 \%$ de los trabajadores desconocen la existencia de normas para el manejo de los desechos hospitalarios, $8 \%$ aseguran que 
no existen, $12 \%$ refieren que sólo se las han comunicado verbalmente, $26 \%$ saben que si existen pero que estas no están disponibles para su revisión y el 33\% refirieron que si existen y que están accesibles para su consulta, lo que crea una gran deficiencia en el conocimiento de los desechos sólidos hospitalarios y su manejo dentro de la institución ya que no es del conocimiento de todos la existencia de las normas dentro de la institución y como obtenerlas. Los resultados se muestran en la gráfica 1 :

Gráfica 1 Normas referentes a la gestión de desechos sólidos hospitalarios en los C.A.P.

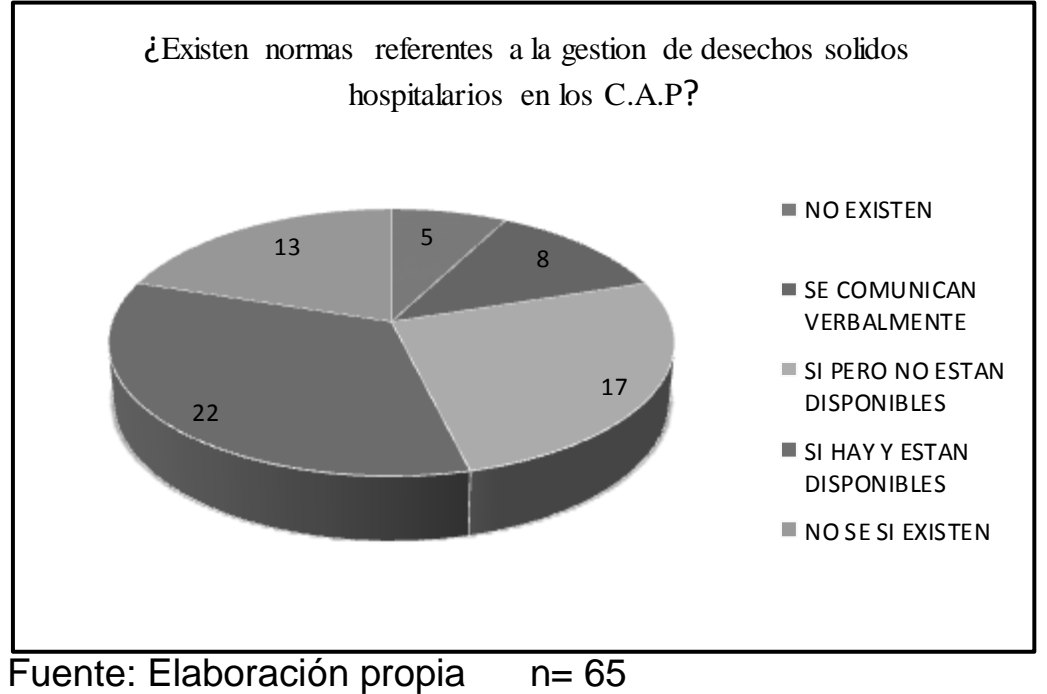

En cuanto al conocimiento de la existencia del Reglamento para el manejo de los desechos sólidos hospitalarios, el Acuerdo Gubernativo 509-2001 en el cual se regula y establece la manera en que deben ser manejados dichos desechos y que es obligación de todo trabajador de salud pública conocerlo, se constató que un $28 \%$ del personal no tiene conocimiento de su existencia como se observa en la gráfica 2 :

Grafica 2 Conocimiento de la existencia del Acuerdo Gubernativo 509-2001: Reglamento para el Manejo de Desechos Sólidos Hospitalarios

¿Sabe usted que existe el reglamento para manejo de desechos solidos hospitalarios, acuerdo gubernativo 509-2001?

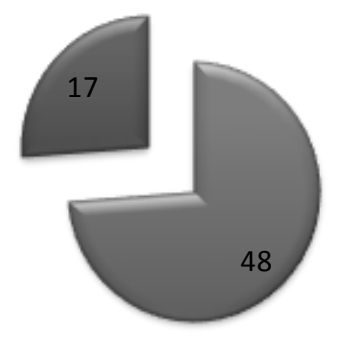

Fuente: Elaboración propia. $\quad \mathrm{n}=65$ 
Para establecer si se está realizando inducción al tema del manejo de desechos sólidos hospitalarios al momento de ingresar a trabajar en los centros de atención, se concluyó que menos de la mitad de los trabajadores refieren haber recibido capacitación al ingresar por primera vez al CAP como se muestra en la gráfica 3 :

Gráfica 3 Capacitaciones sobre el manejo de desechos sólidos hospitalarios al momento de su ingreso al CAP

$\mathrm{Al}$ momento de su ingreso al centro de atención permanente ¿le dieron capacitación sobre el menejo de desechos sólidos hospitalarios?

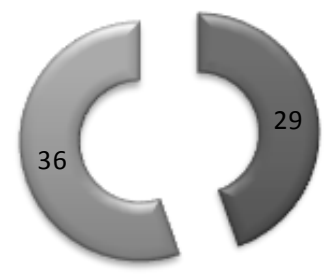

$\square \mathrm{SI} \square \mathrm{NO}$

Fuente: Elaboración propia

Al entrevistar si los trabajadores han recibido capacitación sobre el manejo de desechos sólidos hospitalarios en otra institución diferente al área de salud de Jalapa se estableció que el $33 \%$ ha recibido capacitación fuera del área de salud,

$$
\mathrm{n}=65
$$

especialmente el personal de enfermería quienes refieren que durante su capacitación en la escuela de enfermería se incluye el tema como contenido de aprendizaje 0 en trabajos anteriores. Los resultados se muestran en la gráfica 4:

Gráfica 4 Capacitaciones sobre el manejo de desechos sólidos hospitalarios fuera del C.A.P.

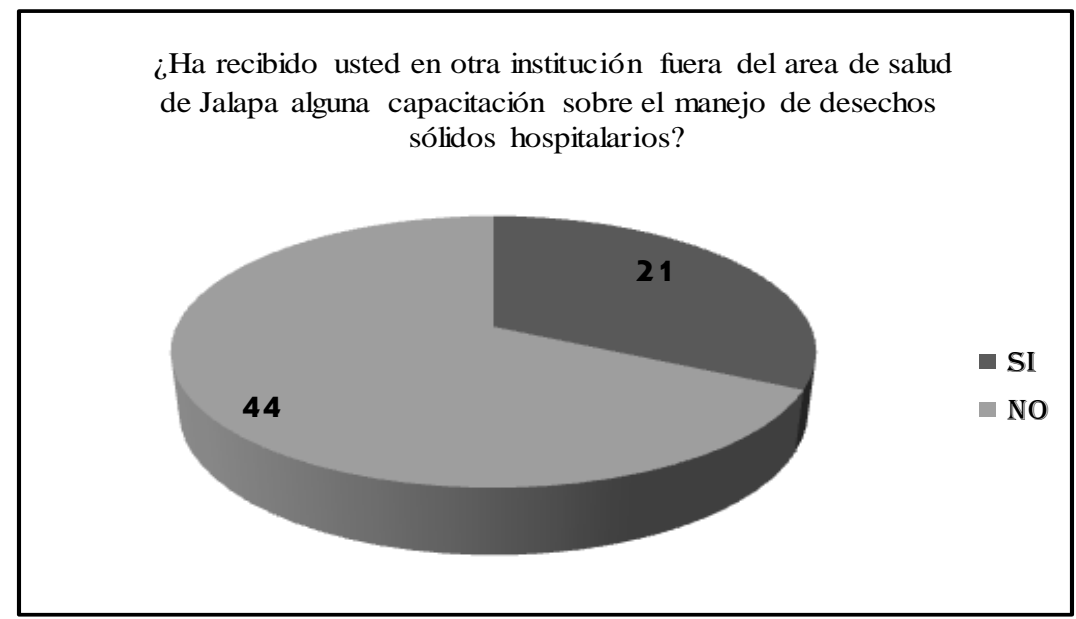

Fuente: Elaboración propia $\quad n=65$

De los 53 trabajadores que contestaron que si conocen el proceso de clasificación de los desechos sólidos hospitalarios se pudo comprobar que más de la mitad no saben que los desechos sólidos se clasifican en desechos bioinfecciosos, especiales y comunes, y que un pequeño porcentaje decidió no contestar a esta pregunta por lo 
cual se confirma que la mayoría de trabajadores cree conocer dicha clasificación pero que en realidad sólo han escuchado al respecto pero no pueden definir dicha clasificación lo cual hace que la posibilidad de contaminación sea mayor. Los resultados de se observan en la gráfica 5 :

Gráfica 5 Clasificación de los desechos sólidos hospitalarios en los C.A.P.

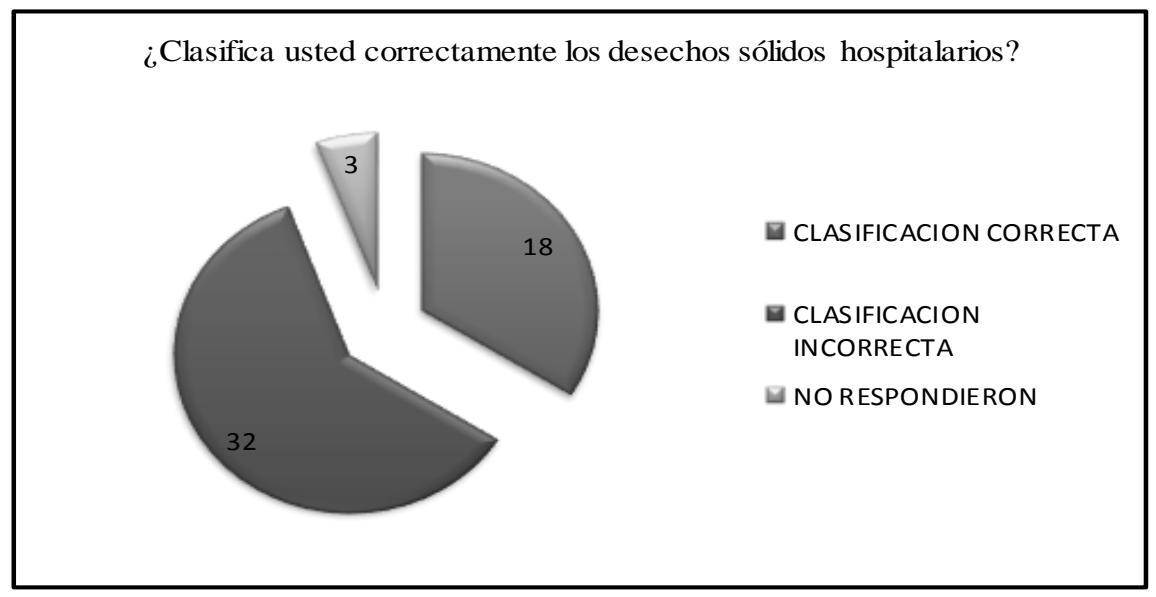

Fuente: Elaboración propia

$\mathrm{n}=65$

Al preguntar a los entrevistados si separan los desechos sólidos bioinfecciosos de los desechos sólidos comunes colocándolos en recipientes específicos únicamente el $9 \%$ refiere que no los colocan en recipientes separados como se refleja en la gráfica 6 :

Gráfica 6 Colocación de los desechos sólidos bioinfecciosos en recipientes separados de los desechos sólidos comunes

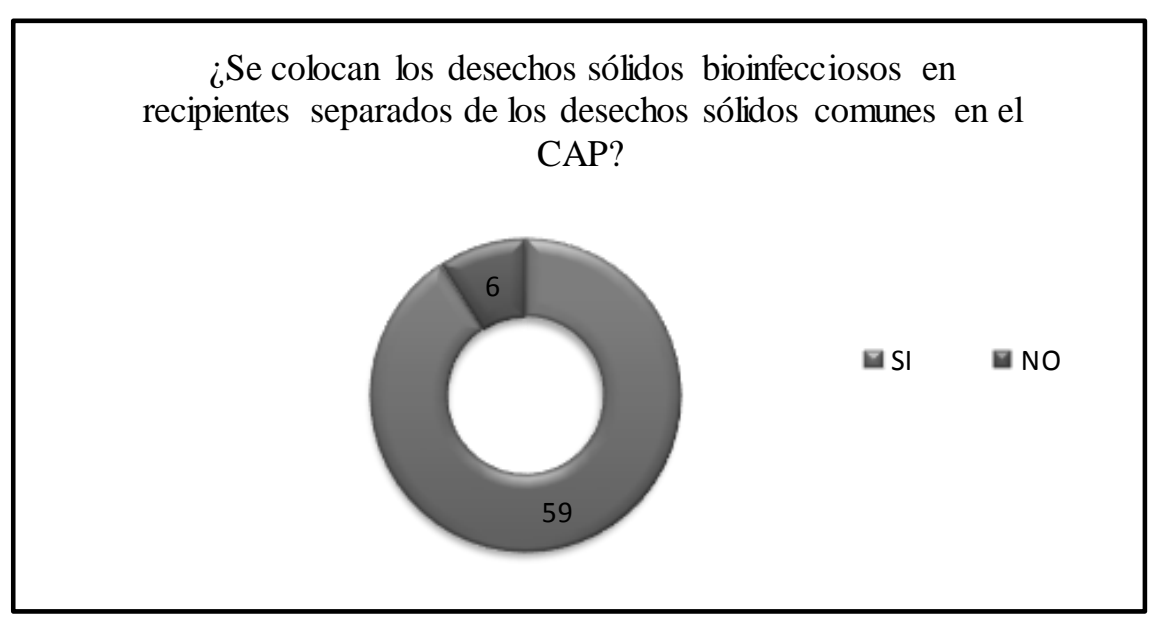

Fuente: Elaboración propia

$$
n=65
$$

Las normas de clasificación de los desechos sólidos hospitalarios indican que los recipientes en los cuales se colocan los desechos deben estar claramente rotulados e identificados para que todas las personas puedan saber dónde se colocan los desechos y clasificarlos adecuadamente, al preguntar si los recipientes de los desechos sólidos bioinfecciosos están claramente rotulados, el $52 \%$ de los entrevistados mencionaron que sí, como se muestra en la gráfica 7 : 
Gráfica 7 Rotulación de los recipientes que se utilizan para los desechos sólidos bioinfecciosos

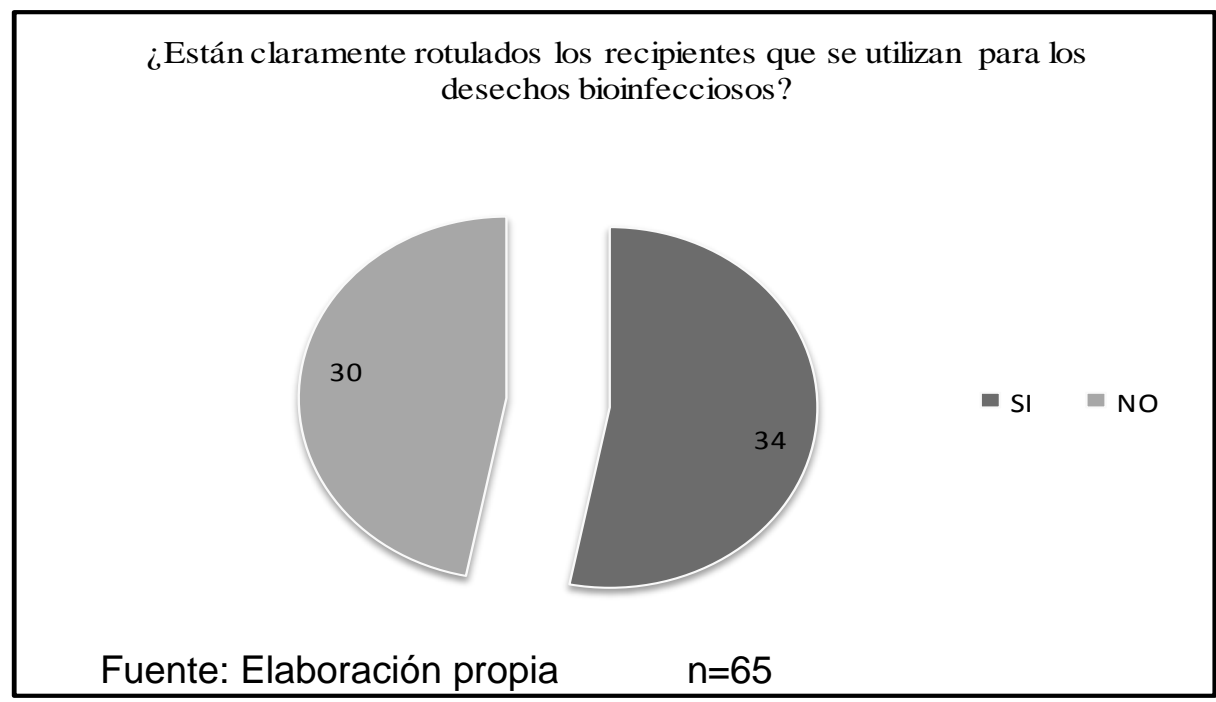

Los desechos contaminados deben ir en recipientes diferentes de los comunes, por lo que se les preguntó a los trabajadores cuál es la diferencia de los recipientes, y el $55 \%$ respondieron que si están claramente rotulados y con bolsas adecuadas, el $41 \%$ respondieron que no tienen rótulos que los identifique y un $3 \%$ indicaron que no se usan recipientes separados. Los resultados se muestran en la gráfica 8:

\section{Gráfica 8 Diferencia entre los recipientes de desechos contaminados de los recipientes de desechos sólidos comunes}

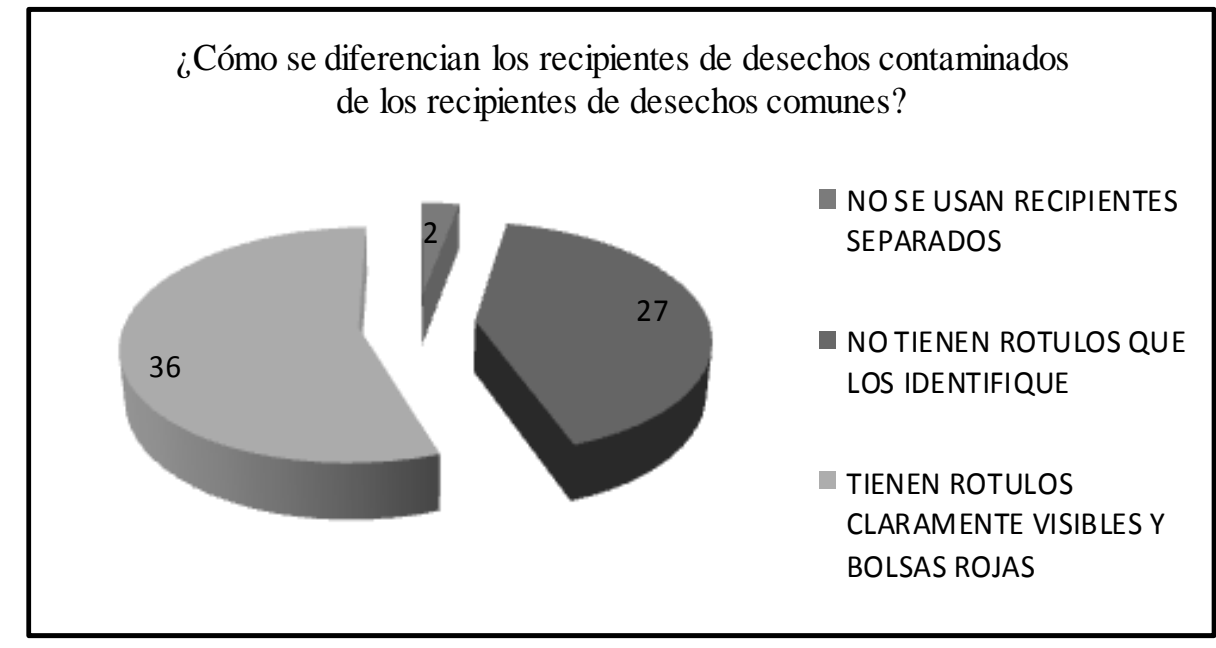

Fuente: Elaboración propia

Los desechos sólidos hospitalarios para su segregación adecuada deben ser colocados en bolsas de colores rojas, negras o blancas dependiente el tipo de desecho, al preguntar sobre dicha clasificación se pudo comprobar $\mathrm{n}=65$

que 17 trabajadores no conocen cómo clasificar los desechos en las diferentes bolsas y que 2 personas decidieron dejar en blanco dicha pregunta. Los resultados se describen en la gráfica 9: 
Gráfica 9 Clasificación de los desechos sólidos hospitalarios según el color de bolsa en que deben ser colocados.

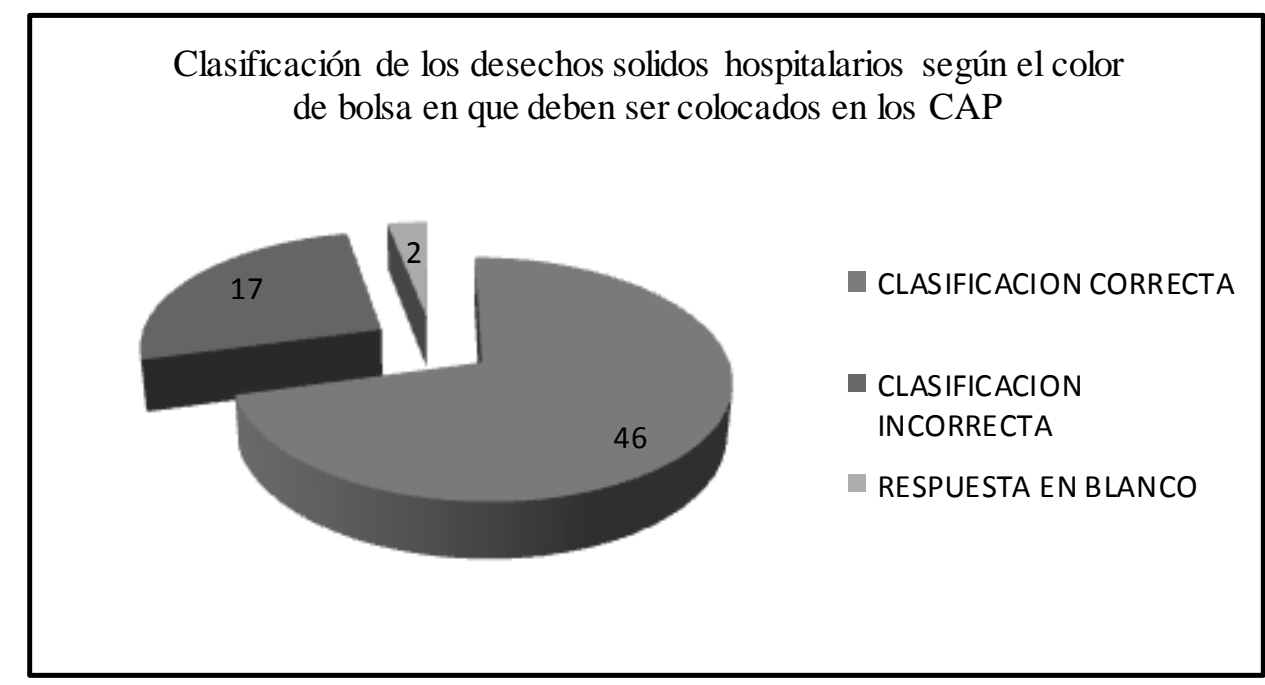

Fuente: Elaboración propia

$\mathrm{n}=65$

En la pregunta sobre si se cuenta en los lugares de trabajo con recipientes adecuados para colocar los desechos punzocortantes 53 personas respondieron que si cuentan con ellos, 12 personas respondieron que no cuentan con dichos recipientes, pero que estos no son necesarios en el lugar donde ellos se encuentran trabajando. Los resultados se muestran en la gráfica 10:

Gráfica 10 Contenedores para los desechos punzocortantes en el lugar de trabajo

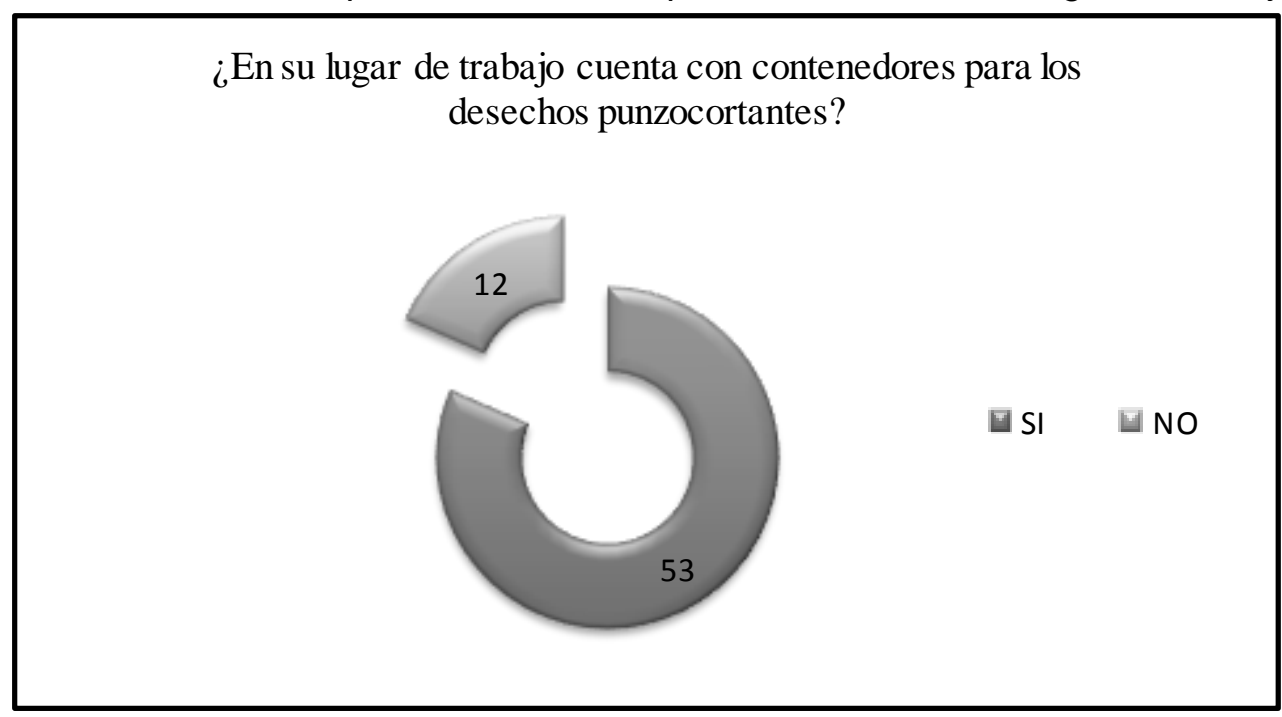

Fuente: Elaboración propia

Las personas que trabajan en el laboratorio confirmaron que si cuentan con equipo

$$
\mathrm{n}=65
$$

adecuado para descartar los desechos bioinfecciosos que ellos manejan como se describe en la gráfica 11: 
Gráfica 11 Equipo adecuado para descartar materiales contaminados con secreciones, fluidos o líquidos humanos en el laboratorio

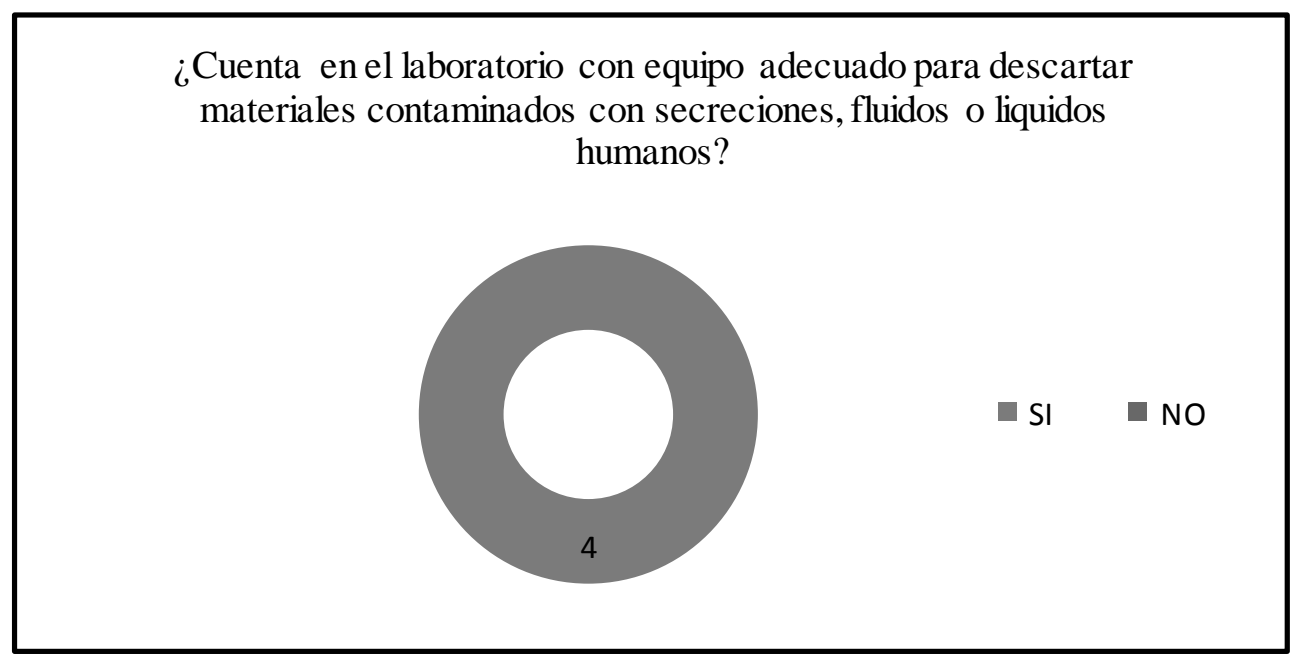

Fuente: Elaboración propia

También se realizaron preguntas al personal operativo de los C.A.P., quienes son los encargados de la manipulación directa de los desechos sólidos hospitalarios más que en la generación de ellos y es el personal más expuesto a contaminación. Se les preguntó a los trabajadores operativos si se les $n=4$

proporciona materiales para protegerse al momento de manipular los desechos sólidos, es decir si cuentan con mascarillas, guantes, uniformes adecuados, respondiendo especialmente que algunas veces se les proporciona pues, no siempre se cuenta con insumos apropiados. Los resultados se presentan en la gráfica 12:

Gráfica 12 Personal operativo con equipo adecuado para el manejo de los desechos sólidos hospitalarios.

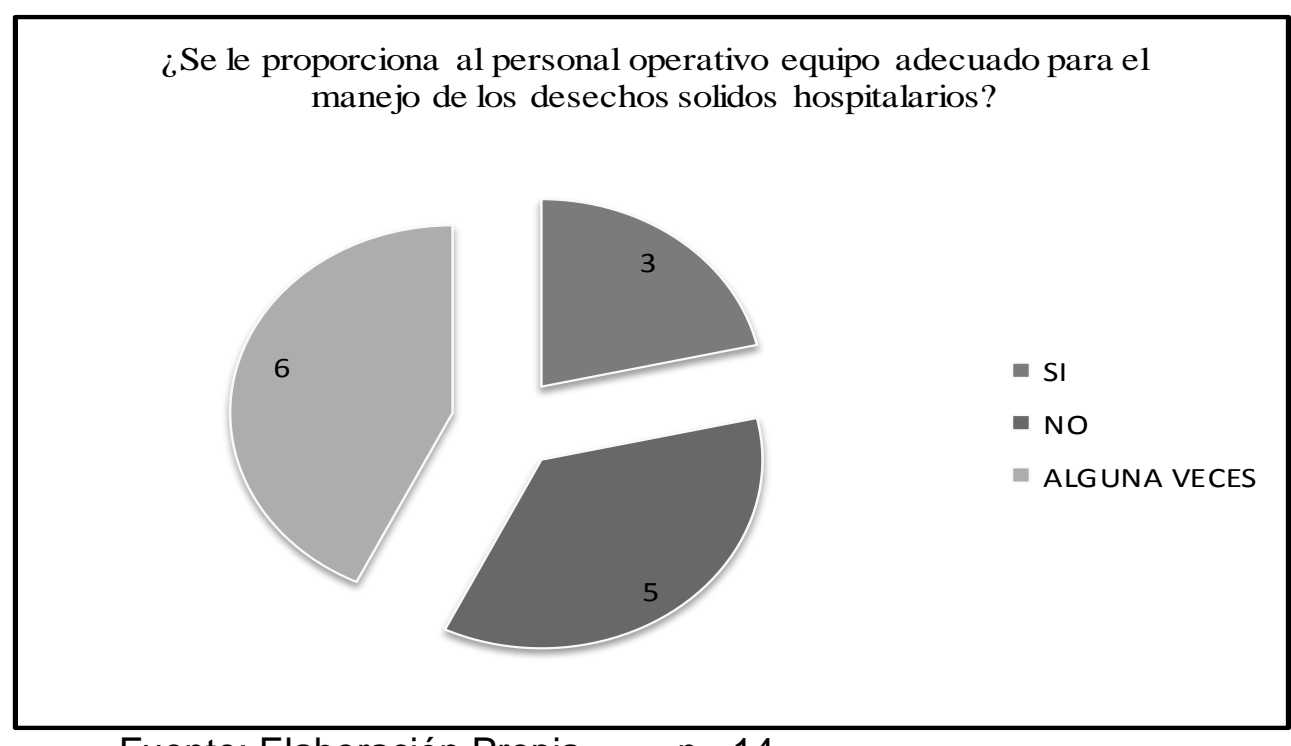

Fuente: Elaboración Propia $\quad \mathrm{n}=14$ 
Los desechos sólidos hospitalarios deben ser embalados adecuadamente para que el ente tratante final pueda darles el manejo apropiado, por lo cual se le preguntó al personal operativo que es el encargado de realizar dicho procedimiento, si hacían el embalaje adecuadamente, por lo que contestaron 6 de ellos que no realizaban el embalaje y el resto si lo realiza, aunque no completo. Los datos se muestran en la gráfica 13:

Gráfica 13 Embalaje de los desechos sólidos hospitalarios

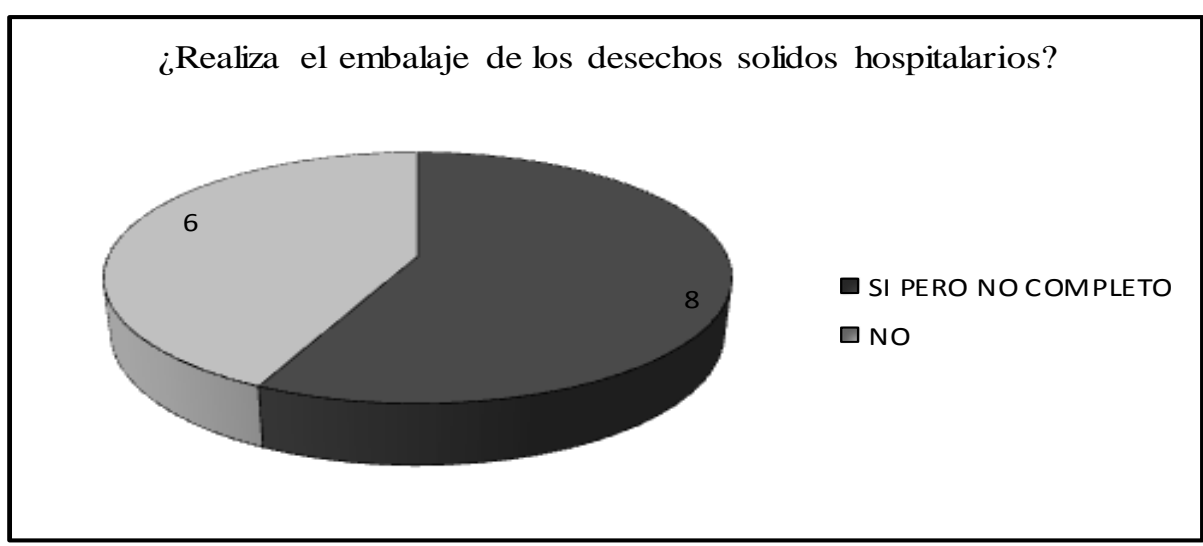

Fuente: Elaboración Propia

$$
\mathrm{n}=14
$$

Respecto a los desechos comunes son tratados junto con la basura municipal lo cual permite que se le dé un tratamiento adecuado sin mezclarlos con los desechos bioinfecciosos como se muestra en la gráfica 14:

Gráfica 14 Tratamiento de los desechos comunes

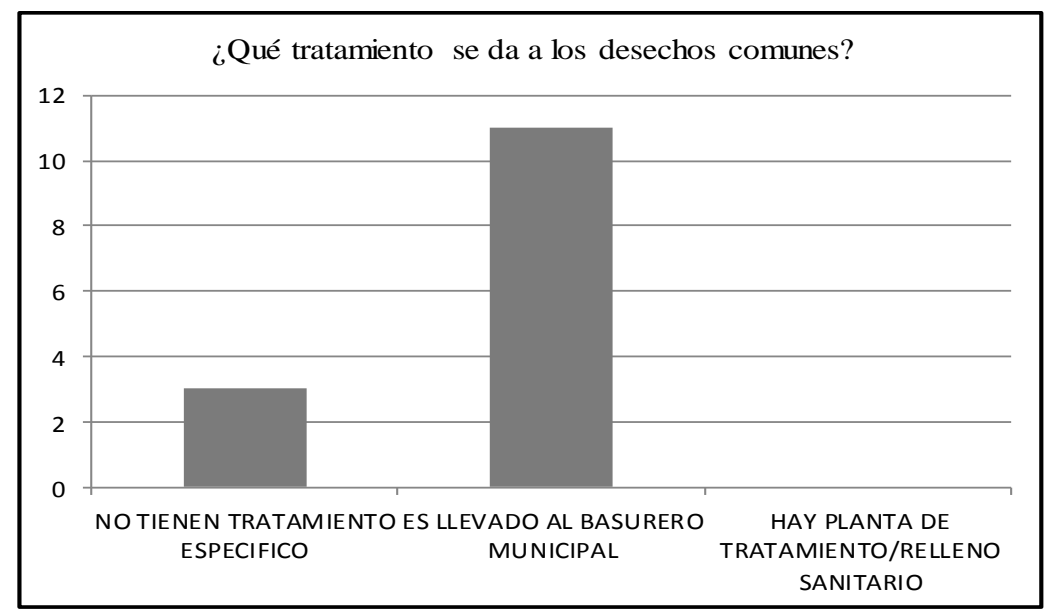

Fuente: Elaboración Propia

Por su parte cuando se preguntó sobre el tratamiento que se le da a los desechos sólidos bioinfecciosos y si dicho tratamiento se hace dentro de la institución o fuera de ella, todo el personal respondió que lo realizan en una planta especial fuera de la institución y que esta es una empresa privada la que se encarga de hacerlo. Este procedimiento se está realizando desde hace un 1 año ya que antes los desechos se acumulaban dentro del servicio en un centro de acopio por no tener un lugar donde se les diera tratamiento. Los resultados se muestran en la gráfica 15: 
Gráfica 15 Tratamiento de los desechos sólidos bioinfecciosos y ente encargado del tratamiento

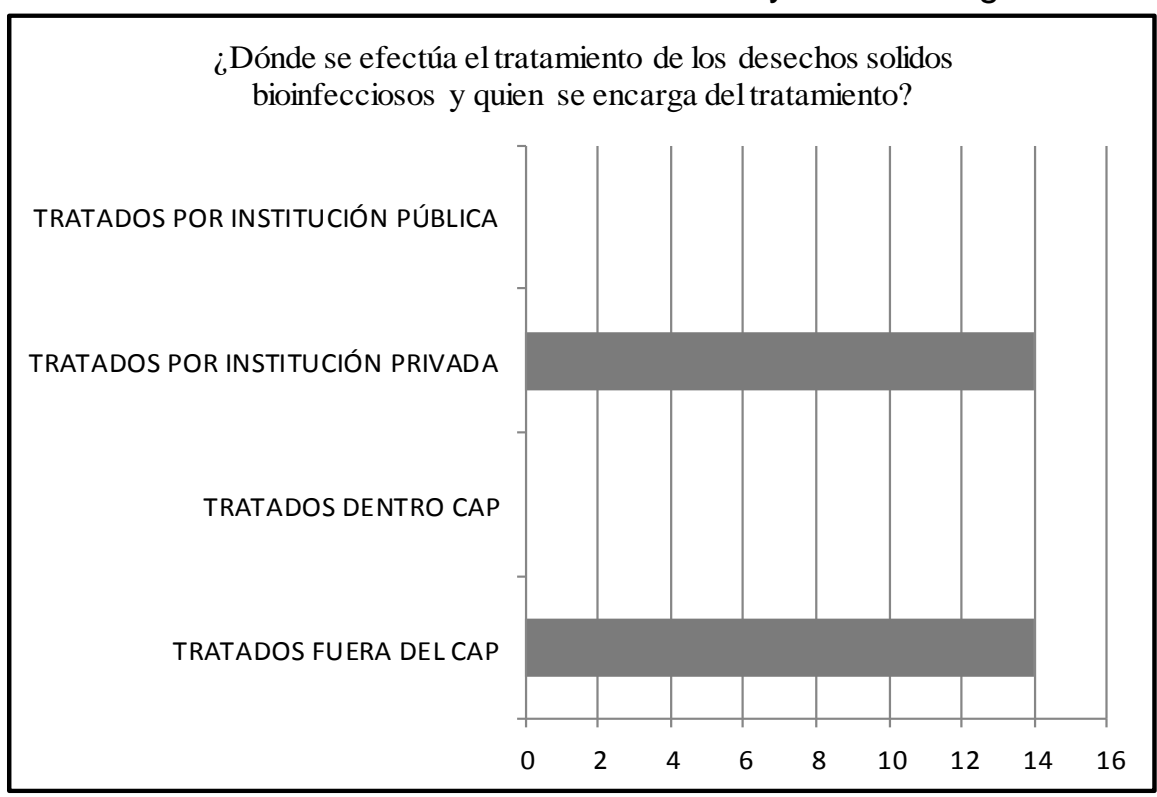

Fuente: Elaboración Propia

Finalmente, al preguntar con qué frecuencia pasan recogiendo los desechos sólidos hospitalarios respondieron que en el Centro Atención Permanente de Mataquescuintla pasan cada 8 días, en el Centro de Atención Permanente de San Carlos Alzatate son ellos mismos los que van a dejar cada 8 días al centro de Salud de Monjas ya que la empresa que le da el tratamiento final no llega hasta dicho Centro y el en Centro de Atención Permanente de Sanyuyo y San Pedro Pínula los llevan al Centro de Salud de Jalapa, sólo que Sanyuyo lo realiza cada 15 días y San pedro Pínula cada 8 días. Los resultados se describen en la gráfica 16 :

Gráfica 16 Frecuencia de la extracción de los desechos sólidos hospitalarios para su tratamiento final

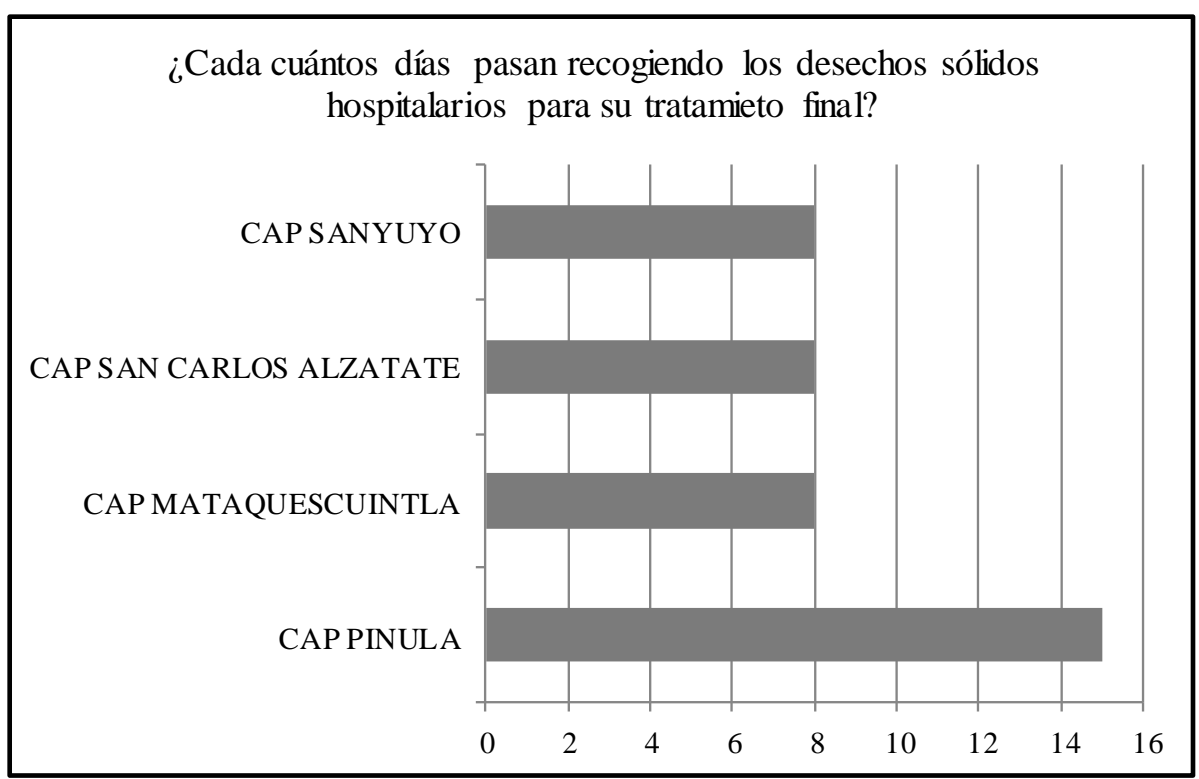

Fuente: Elaboración Propia 


\section{Discusión de resultados}

De acuerdo a los resultados obtenidos, se confirmó que el tema de educación ambiental no se ha estado impartiendo en los Centros de Atención Permanente y que las personas entrevistadas no relacionan el manejo de desechos sólidos hospitalarios como tema de educación ambiental.

Se ha verificado que no todos los trabajadores de los CAP conocen el acuerdo Gubernativo 509-2001 el cual trata sobre el reglamento para el manejo de desechos sólidos hospitalarios, y aunque el tema ha sido impartido alguna vez, no se ha realizado de forma continua por lo cual hay personal que aún no ha sido capacitado, y no recibieron inducción sobre el tema al momento de su ingreso al Centro de Atención Permanente ni en otras instituciones donde estuvieron previamente. Mucha de la información se está trasladando de forma verbal, es decir que lo que un trabajador sabe lo hace llegar a otro y se copian o se heredan las formas de manejar los desechos sólidos hospitalarios sin realmente tener una base teórica al respecto.

Con respecto a la clasificación de los desechos sólidos hospitalarios, aunque la mayoría de los trabajadores indican conocer la clasificación, al momento de preguntarles directamente no lo pudieron explicar por lo que se comprueba la falta de conocimiento y la necesidad de reforzamiento.

Una de las principales manifestaciones que se hicieron al momento de la entrevista y de la capacitación fue que no se cuenta con los recursos adecuados para poder colocar los desechos sólidos hospitalarios en los recipientes correspondientes, que por ejemplo en algunos momentos determinados se cuentan con las bolsas rojas, negras y blancas utilizándose adecuadamente, pero cuando no se tienen, que es la mayoría de veces, se busca otra manera de poder clasificarlas, encajas, botes o bolsas de un solo color pero rotulándolas, y cuando hay bolsas de colores no tienen el grosor adecuado para que realmente puedan cumplir con los requisitos que se determinan por el reglamento de manejo de desechos sólidos hospitalarios.

Al comprobar que no se cuenta con los recursos adecuados para la clasificación de los desechos sólidos hospitalarios se está haciendo un mal manejo de los mismos aunque se tenga la intención. Otra información importante es que la empresa encargada del tratamiento final de los desechos generados en los Centros de Atención Permanente no pasa directamente a todos los establecimiento debiéndose de trasladar a segundos lugares en transportes que no son los apropiados lo cual hace que el tren de aseo no sea el más seguro. Por lo que las personas encargadas de la manipulación están más expuestas a contaminarse.

\section{Conclusiones}

La educación ambiental no es un tema que se esté impartiendo en forma continua ni estructurada dentro de los Centros de Atención Permanente, a pesar que el Ministerio de Salud es uno de los encargados de velar por el mantenimiento del equilibrio ecológico y del medio ambiente a través del adecuado manejo de los desechos sólidos hospitalarios.

El Acuerdo Gubernativo 509-2001 "Reglamento para el Manejo de desechos sólidos Hospitalarios" es conocido por muy pocos trabajadores de los CAP del departamento de Jalapa, por lo cual es necesario que se divulgue dicho Acuerdo para el adecuado manejo de los desechos sólidos hospitalarios y así evitar la contaminación de los trabajadores, asistentes a los servicios de salud y de la población en general.

El personal de los Centros de Atención Permanentes en Jalapa no está cumpliendo las indicaciones del Ministerio de Salud Pública y Asistencia Social sobre el manejo de desechos sólidos hospitalarios debido al desconocimiento de las normas para su manejo y por falta de insumos necesarios 
para poderlos clasificar y descartar adecuadamente.

El personal operativo de los CAP de Jalapa son los que se encargan de manejar los desechos sólidos hospitalarios llevándolos a los diferentes centros de acopio, determinándose que son ellos los que menos conocimiento tienen sobre el adecuado manejo de los desechos en mención.

La falta de los insumos necesarios para poder clasificar y almacenar adecuadamente los desechos sólidos hospitalarios es un problema vital por resolver ya que los trabajadores utilizan alternativas con los recursos que tienen, pero no se cumplen los requisitos mínimos que el Ministerio de Salud pide para el manejo de dichos desechos.

Al momento de ingresar a trabajar en los Centros de Atención Permanente del área de Jalapa no se realiza una inducción al personal sobre cómo manejar los desechos sólidos hospitalarios, y la importancia que estos tienen para proteger el medio ambiente y la salud de la población en general.

Dentro de los Centros de Atención Permanente en estudio excepto en Sanyuyo existen lugares cerrados que utilizan como centros de acopio para los desechos sólidos pero no se están almacenando adecuadamente $y$ no se cumplen los requisitos necesarios.

\section{Referencias}

Área Metropolitana de Valle de Aburrá (2008)

Manual para el Manejo Integral de Residuos en el Valle de Aburrá. Colombia.

Arze, M. et al. Manual de Manejo de Residuos, Bioseguridad, y Prevención de Infecciones Nosocomiales, Instuto Nacional de Oftalmología “Javier Pescador Sarget" La Paz, Bolivia.

Benenson, A. (1995) "Control of communicable diseases manual". 16th edition. Washington, American Public Health Association.
Carranza, J. (2002) "Evaluación y recomendaciones el manejo de residuos hospitalarios en un centro de asistencia de salud del área norcentro de la ciudad de Guatemala" conferencia dictada en XXVIII Congreso Interamericano de Ingeniería Sanitaria y Ambienta Cancún, México, 27 al 31 de octubre de 2002.

De León, D. (2011). Proyecto de Factibilidad para la Implementación de Un sistema de Tratamiento de desechos sólidos hospitalarios en el Hospital Roosevelt de la ciudad de Guatemala. Guatemala, Facultad de Ingeniería, Escuela de Ingeniería Mecánica Industrial. USAC.

Díaz, C. (2007) Plan de Gestión de Residuos Servicio Andaluz de salud, Dirección General de Asistencia Sanitaria, España.

Dirección de Área de Salud de Jalapa (2006) Manual de Organización, Procedimientos y Normas del Personal de Salud, Jalapa.

García, J. (2010) "Diagnóstico del sistema de manejo de desechos sólidos generados en el Hospital "Dr. Julio Criollo Rivas" revista Salud de los trabajadores, volumen 18 No. 1 Maracay junio 2010.

Gualdron, S. y M. Calderón, (2012) Plan de Gestión integral de Residuos Hospitalarios y similares para la Universidad de Santander UDES Verde, Campus Lagos del Cacique Bucaramanga Bogotá.

Hernández, E. et al (2006) Intervención educativa para incrementar los conocimientos sobre bioseguridad en el personal de enfermería de una Institución hospitalaria. Revista Cubana de Enfermería, Vol. 22 No.2.

INS (Instituto Nacional de Salud) (2010) Manual de Gestión Integral, subdirección Red Nacional de Laboratorios República de Colombia, Mayo 2010.

ISL (Instituto de Salud Laboral) (2011) Manejo de Residuos Hospitalarios Curso 12. 1ra edición, Santiago de Chile, Ministerio de trabajo y Previsión social, Julio 2011.

Junco, A., et al. (2000) "Desechos hospitalarios: aspectos educativos en la implementación de su manejo" Revista Cubana de Higiene y Epidemiología, Volumen 38 No. 3 Ciudad de la Habana Mayo-agosto. 2000, pp 195-200. 
Junco, A., et al. (2000) "Desechos Hospitalarios: Aspectos Metodológicos de su Manejo" Instituto Nacional de Higiene, Epidemiologia y Microbiología. Revista Cubana Higiene y Epidemiologia 2000 volumen 38 No. 2 pp 122126.

Katoch, S., (2007) "Clasificación de Residuos Biomédicos y de Gestión Predominante de Estrategias" Actas de la Conferencia Internacional sobre la Gestión Sostenible de Residuos Sólidos 5 a7 septiembre, Chennai, India. pp. 169-175

Koning, K. et al., (1994) Desechos peligrosos y salud en América Latina y El caribe, Programa Regional de la Organización Panamericana de la Salud, Washington, D.C.

Lacayo, M. (2008) La Gestión Integral de los Residuos en Centroamérica, el Caso de Nicaragua. In: Andrés, P y Rodríguez, R. (Eds) Documenta Universitaria. Girona, España, pp. 267-283.

Larrañaga, E. y A. Fernández. (2012) Guías de Prevención de Infecciones Hospitalarias Casa de Galicia 2ª edición Montevideo, 2012

Leal, M. et al (2004) “ Manejo de desechos peligrosos en los establecimientos de salud del área Alajuela Oeste" Revista Costarricense de Salud vol.13 no.24 San José Costa Rica, Julio 2004.

Llorente S., et al (1997) "Evaluación de la gestión Hospitalaria de Residuos sanitarios en el Principado de Asturias." Servicio de Inspección de Salud, Consejería de Servicios sociales del Principado de Asturias. Revista Española de Salud Pública volumen 71 no .2 Madrid Mar./Abr. Pp 189-199.

Mata. S, et al (2006) Programa de Educación Ambiental para La Gestión de los Desechos Hospitalarios. Revista Universidad, Ciencia y Tecnología. Volumen 10, № 41, diciembre 2006, pp. 286-300

Ministerio de Salud de Chile, (2001) Desechos Hospitalarios: Riesgos Biológicos y Recomendaciones Generales Sobre su Manejo División de inversiones y desarrollo de la Red asistencial departamento de calidad en la red unidad de evaluación de tecnologías de salud, Chile Agosto 2001.

Ministerio de Salud Y Ministerio de ambiente de Colombia (2002) Manual de Procedimientos para la gestión integral de residuos hospitalarios y similares en Colombia MPGIRH 2002.

MSPAS (Ministerio de Salud Pública y Asistencia social) (2001) Reglamento para el manejo de desechos hospitalarios acuerdo gubernativo5092001. Guatemala. MSPAS.

MSPAS (Ministerio de Salud Pública y Asistencia Social). (2014) "Niveles de atención de Salud" Portal de Información del ministerio de Salud de Guatemala, disponible en: http://portal.mspas.gob.gt/index.php/en/segundonivel-de-atencion.html [consultado el 12 enero 2014]

Mulet. C., I Castanedo (2002) "La Participación Comunitaria y el medio ambiente". Revista Cubana de Enfermería. Volumen 18 No. 2 Ciudad de la Habana, abril-junio. 2002.

Neveu, A. y P. Matus. (2007) "Residuos hospitalarios peligrosos en un Centro de Alta Complejidad" revista médica Chile 2007; volumen 135 pp 885-895.

ONU, (1992) Declaración de Rio Sobre el Medio Ambiente y el Desarrollo, Conferencia de las Naciones Unidas sobre el Medio Ambiente y el Desarrollo. Rio de Janeiro, Brasil.

OMS (2002) Prevención de las infecciones nosocomiales guía práctica segunda edición WHO/CDS/CSR/EPH/2002.

OPS (Organización Panamericana de la Salud) (2007) Perfil de los Sistemas de Salud Guatemala, Monitoreo y Análisis de Los Procesos de Cambio y Reforma, Tercera Edición, Guatemala.

Palma, E. (2008) Diseño de un Programa de Capacitacion sobre riesgo biológico y desechos de manejos solidos hospitalarios tipo C dirigido al personal de enfermería que labora en las unidades de diálisis, Barquisimeto, estado Lara. Trabajo presentado para obtener el grado de especialista en Salud e Higiene Ocupacional Universidad Centro Occidental Lisandro Alvarado. Venezuela.

Plaza, G. y O. Zapata (2011) "Residuos y Salud: Tartagal-Salta" Revista de Ciencia y Tecnología No. 16 Julio/diciembre 2011 
Pruss, A., et al (1999). Safe management of wastes from health-care activities. World Health Organization Geneva.

Ramírez, J. (2005). La disposición final de los desechos sólidos hospitalarios y factibilidad de instalación de un incinerador en el Hospital regional de occidente "San Juan de Dios" de Quetzaltenango. Guatemala, Facultad de Ingeniería, Escuela de Ingeniería Mecánica. USAC.

Rodriguez, G. y G. Trindade (2010) Gestión Integral de Residuos Hospitalarios. Centro Nacional de Quemados, Uruguay, noviembre del 2010.

Rodríguez, M. (2006) El manejo de los desechos sólidos en Guatemala. Guatemala, Facultad de Humanidades Maestría en Docencia Universitaria. USAC.

Ruiz, M., (2012) "Caracterización de Residuos Sólidos en la Universidad Iberoamericana, ciudad de mexico" En Revista Internacional de Contaminación Ambiental, Volumen 28 No. 1, pp. 93-97 2012

Salud sin Daño (2007) "Residuos Hospitalarios" Guia para reducir su impacto sobre la Salud y el ambiente, Segunda edición. Octubre 2007

Sardiñas, O., et al (2001) "Exposición de riesgo para la salud por exposición a residuos peligrosos" Revista Cubana de Higiene y epidemiologia volumen 39 No. 2 ciudad de la Habana mayoragosto, pp 144-146.

Suarez, P., R., Junco (2012) "Plan institucional de manejo de los desechos sólidos, una herramienta para la gestión hospitalaria" Revista Cubana de higiene y epidemiología Volumen 40, No. 3, pp. 415-419.

URL (Universidad de Rafael Landivar) et al (2003) Generación y Manejo de Desechos Sólidos en Guatemala. Instituto de Incidencia Ambiental, Guatemala, agosto de 2003.

UTP (Universidad Tecnológica de Pereira) (2006) Plan de gestión Integral Residuos Hospitalarios y similares. Pereira, Abril de 2006.

Vásquez, O., (2011) "Gestión de los residuos sólidos municipales en la ciudad del Gran Santiago de Chile: desafíos y oportunidades "Revista internacional de contaminación ambiental, volumen 27 No. 4 México, noviembre 2011.

Zambrano, A. (2009) Educación ambiental: La teoría y Práctica para el mejoramiento Urbano. Serie: Instrumentos para la Gestión ambiental Urbana, No. 3 Fundación Natura, Quito Ecuador. 2009 
